\title{
A short overview of MRI artefacts
}

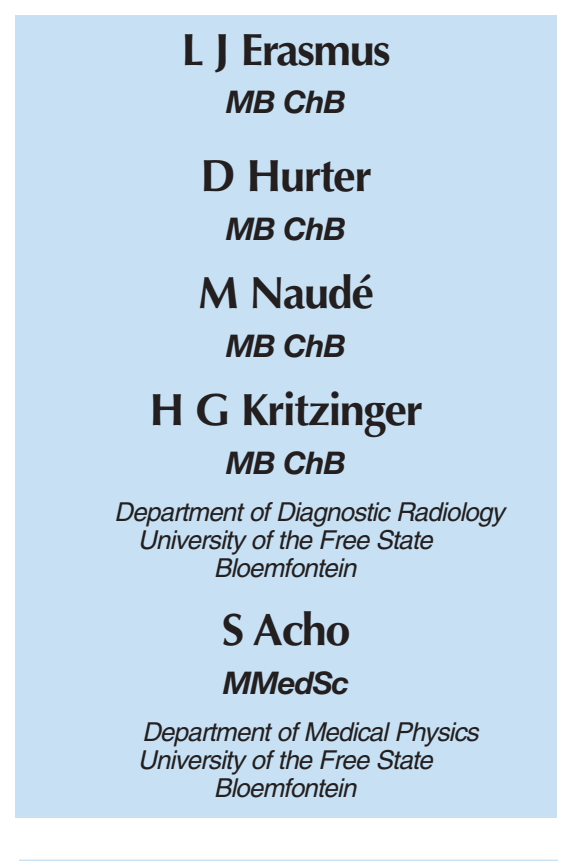

\section{Abstract}

Many different artefacts can occur during magnetic resonance imaging (MRI), some affecting the diagnostic quality, while others may be confused with pathology. An artefact is a feature appearing in an image that is not present in the original object. Artefacts can be classified as patientrelated, signal processing-dependent and hardware (machine)-related. This article presents an overview of MRI artefacts and possible rectifying methods.

\section{Introduction}

Artefacts remain a problematic in magnetic resonance imaging (MRI). Some affect the quality of the examination, while others may be confused with pathology.

An artefact is a feature appearing in an image that is not present in the original object. Depending on their origin, artefacts are typically classified as patient-related, signal processingdependent and hardware (machine)related.

It is important to recognise these artefacts and have a basic understanding of their origin, especially those mimicking pathology. In this article emphasis is placed on recognition of artefacts and possible rectifying methods.

\section{Patient-related MR artefacts}

\section{Motion artefacts}

Motion is one of the most common artefacts in MR imaging, causing either ghost images or diffuse image noise in the phase-encoding direction. The reason for mainly affecting data sampling in the phase-encoding direction is the significant difference in the time of acquisition in the frequency- and phase-encoding directions. Frequency-encoding sampling in all the rows of the matrix $(128,256$ or 512) takes place during a single echo (milliseconds). Phase-encoded sampling takes several seconds, or even minutes, owing to the collection of all the $\mathrm{k}$-space lines to enable Fourier analysis. Major physiological movements are of millisecond to seconds duration and thus too slow to affect frequency-encoded sampling, but they have a pronounced effect in the phase-encoding direction.

Periodic movements such as cardiac movement and blood vessel or CSF pulsation cause ghost images, while non-periodic movement causes diffuse image noise (Fig. 1). Ghost image intensity increases with amplitude of movement and the signal intensity from the moving tissue.

Several methods can be used to reduce motion artefacts, including patient immobilisation, ${ }^{1}$ cardiac and respiratory gating, ${ }^{2}$ signal suppression of the tissue causing the artefact, ${ }^{1}$ choosing the shorter dimension of the matrix as the phase-encoding direction, view-ordering or phase-reordering methods ${ }^{3}$ and swapping phaseand frequency-encoding directions ${ }^{1}$ to move the artefact out of the field of interest.

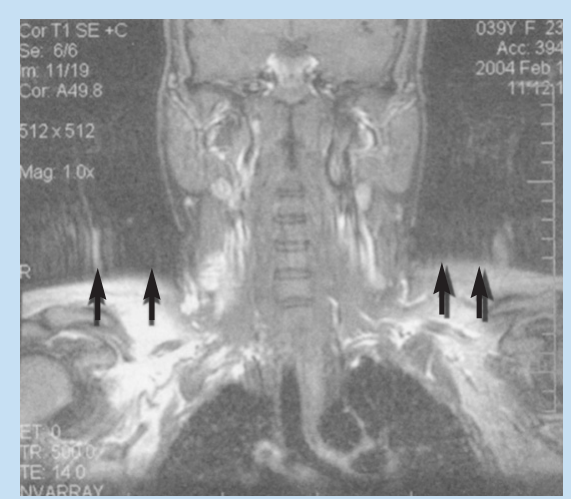

Fig. 1. Motion artefact (T1 coronal study of lumbar vertebrae).

\section{Flow}

Flow can manifest as either altered intravascular signal (flow enhancement or flow-related signal loss), or flow-related artefacts (ghost images or spatial misregistration).

Flow enhancement, also known as inflow effect, is caused by fully magnetised protons entering the imaged slice while the stationary protons have not fully regained their magnetisation. 
The fully magnetised protons yield a high signal in comparison with the rest of the surroundings.

High velocity flow causes the protons entering the image to be removed from it by the time the 180-degree pulse is administered. The effect is that these protons do not contribute to the echo and are registered as a signal void or flow-related signal loss (Fig. 2).

Spatial misregistration manifests as displacement of an intravascular signal owing to position encoding of a voxel in the phase direction preceding frequency encoding by time TE/2. The intensity of the artefact is dependent on the signal intensity from the vessel, and is less apparent with increased TE.

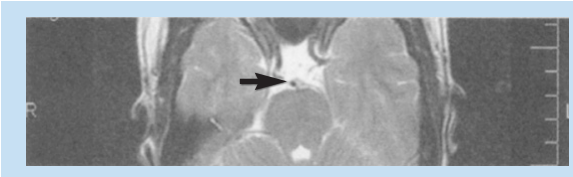

Fig. 2. Flow-related signal loss in the carotid and basillary arteries (T2 axial study of the brain).

\section{Metal artefacts}

Metal artefacts occur at interfaces of tissues with different magnetic susceptibilities, which cause local magnetic fields to distort the external magnetic field. This distortion changes the precession frequency in the tissue leading to spatial mismapping of information. The degree of distortion depends on the type of metal (stainless steel having a greater distorting effect than titanium alloy), ${ }^{4}$ the type of interface (most striking effect at soft tissue-metal interfaces), pulse sequence and imaging parameters.

Metal artefacts are caused by external ferromagnetics such as cobalt containing make-up, or internal ferromagnetics such as surgical clips, spinal hardware and other orthopaedic devices. Manifestation of these artefacts is variable, including total signal loss, peripheral high signal and image distortion (Figs 3 and 4).

Reduction of these artefacts can be attempted by orientating the long axis of an implant or device parallel to the long axis of the external magnetic field, possible with mobile extremity imaging and an open magnet., ${ }^{5,6}$ Further methods used are choosing the appropriate frequency encoding direction, since metal artefacts are most pronounced in this direction, ${ }^{4}$ using smaller voxel sizes, ${ }^{5}$ fast imaging sequences, ${ }^{6}$ increased readout bandwidth $^{5,6}$ and avoiding gradient-echo imaging when metal is present. ${ }^{5} \mathrm{~A}$ technique called MARS (metal artefact reduction sequence) applies an additional gradient, along the slice select gradient at the time the frequency encoding gradient is applied. ${ }^{5}$

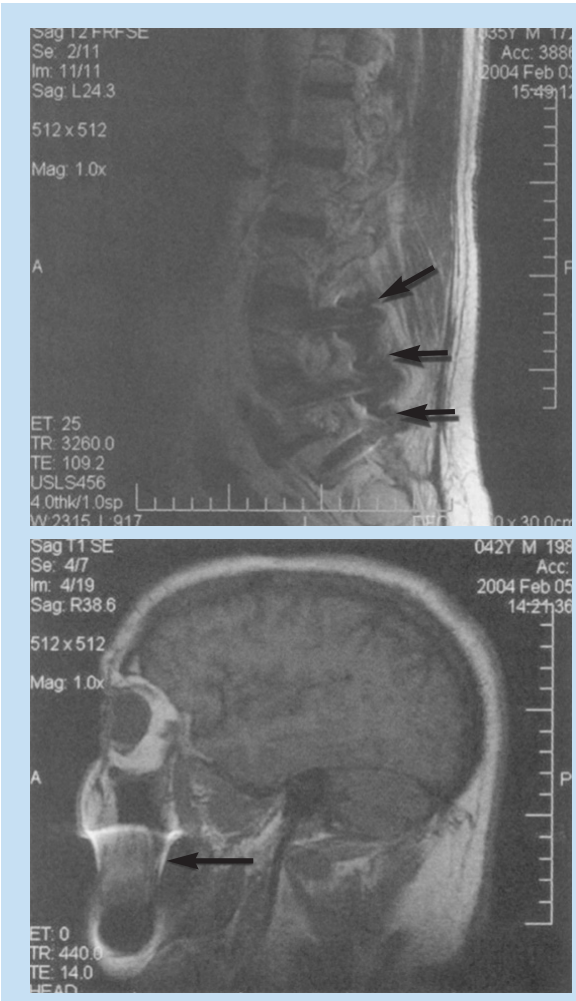

Figs 3 and 4. Metal-related artefacts.

\section{Signal processing- dependent artefacts}

The ways in which the data are sampled, processed and mapped out on the image matrix manifest these artefacts.

\section{Chemical shift artefact}

Chemical shift artefact occurs at the fat/water interface in the phase encoding or section-select directions (Fig. 5). These artefacts arise due to the difference in resonance of protons as a result of their micromagnetic environment. The protons of fat resonate at a slightly lower frequency than those of water. High field strength magnets are particularly susceptible to this artefact.?

Determination of the artefact can be made by swapping the phase- and frequency-encoding gradients and examining the resultant shift (if any) of the tissues.

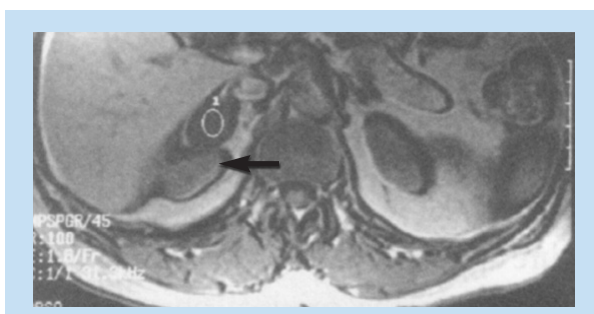

Fig. 5. Chemical shift artefact: bright and dark streaks around the kidneys in an axial gradientecho opposed-phase image (acknowledgement GE Health Care).

\section{Partial volume}

Partial volume artefacts arise from the size of the voxel over which the signal is averaged. Objects smaller than the voxel dimensions lose their identity, and loss of detail and spatial resolution occurs. Reduction of these artifacts is accomplished by using a smaller pixel size and/or a smaller slice thickness. 


\section{Wrap around}

This artefact is a result of mismapping of anatomy that lies outside the field of view but within the slice volume. The selected field of view is smaller than the size of the imaged object. The anatomy is usually displaced to the opposite side of the image (Figs 6 and 7). It can be caused by non-linear gradients or by undersampling of the frequencies contained within the return signal.

The sampling rate must be twice the maximal frequency that occurs in the object (Nyquist sampling limit). If not, the Fourier transform will assign very low values to the frequency signals greater than the Nyquist limit. These frequencies will then 'wrap around' to the opposite side of the image, masquerading as low-frequency signals. In the frequency encode direction a filter can be applied to the acquired signal to eliminate frequencies greater than the Nyquist frequency. In the phase encode direction, artefacts can be reduced by an increasing number of phase encode steps (increased image time). For correction, a larger field of view may be chosen.

\section{Gibbs phenomenon (ring- ing artefact)}

This is caused by the under-sampling of high spatial frequencies at sharp boundaries in the image. ${ }^{8,9}$ Lack of appropriate high-frequency components leads to an oscillation at a sharp transition known as a ringing artefact. The artefact occurs near the sharp boundaries, where high contrast transitions in the object occur. It appears as multiple, regularly spaced parallel bands of alternating bright and dark signal that slowly fade with
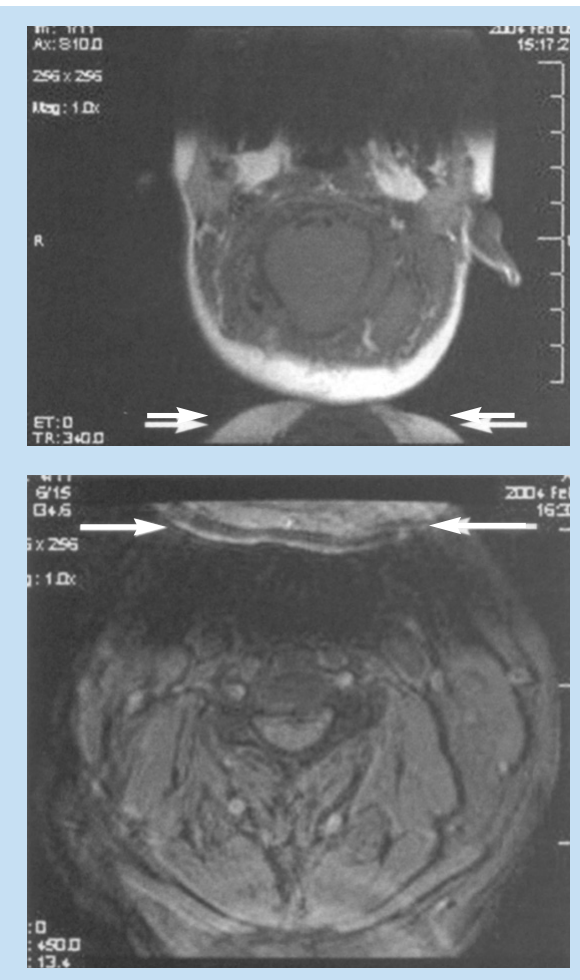

Figs 6 and 7. Wrap around artefacts.

distance (Fig. 8). Ringing artefacts are more prominent in smaller digital matrix sizes.

Methods employed to correct Gibbs artefact include filtering the Kspace data prior to Fourier transform, increasing the matrix size for a given field of view, the Gegenbauer reconstruction and Bayesian approach. ${ }^{10-12}$

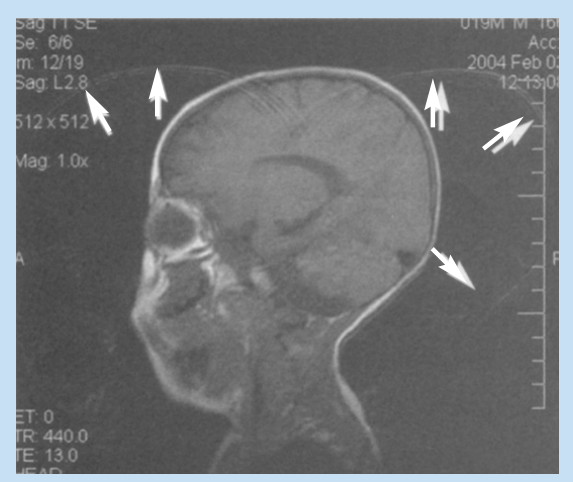

Fig. 8. Gibbs artefact (T1 sagittal study of the brain).

\section{Machine/hard- ware-related artefacts}

This is a wide, still expanding subject and should be discussed separately. Only a few common artefacts that should be recognised are mentioned. When in doubt, however, a technician should be consulted.

\section{Radiofrequency (RF) quad- rature $^{13}$}

RF detection circuit failure arises from improper detector channel operation. Fourier-transformed data display a bright spot in the centre of the image. If one channel of the detector has a higher gain than the other it will result in object ghosting in the image. This is the result of a hardware failure and must be addressed by a service representative.

\section{External magnetic field (B0) inhomogeneity ${ }^{14}$}

B0 inhomogeneity leads to mismapping of tissues. Inhomogeneous external magnetic field causes either spatial, intensity, or both distortions. Intensity distortion occurs when the field in a location is greater or less than in the rest of the imaged object (Fig. 9). Spatial distortion results from long-range field gradients,

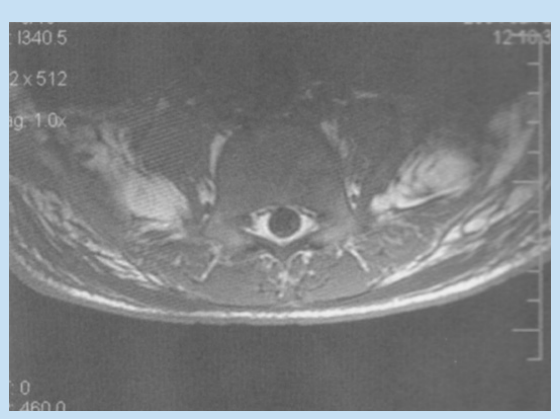

Fig. 9. BO inhomogeneity: intensity distortion across a T1 axial study of the lumbar vertebrae. 
which remain constant in the inhomogeneous field.

\section{Gradient field artefacts (B1 inhomogeneity $)^{15}$}

Magnetic field gradients are used to spatially encode the location of signals from excited protons within the volume being imaged. The slice select gradient defines the volume (slice). Phase- and frequency-encoding gradients provide the information in the other two dimensions. Any deviation in the gradient would be represented as a distortion.

As the distance increases from the centre of the applied gradient, loss of field strength occurs at the periphery. Anatomical compression occurs and is especially pronounced on coronal and sagittal imaging.

When the phase-encoding gradient is different, the width or height of the voxel are different, resulting in distortion. Anatomical proportions are compressed along one or the other axis. Square pixels (and voxels) should be obtained.

Ideally the phase gradient should be assigned to the smaller dimension of the object and the frequency gradient to the larger dimension. In practice this is not always possible because of the necessity of displacing motion artefacts.

This may be corrected by reducing the field of view, by lowering the gradient field strength or by decreasing the frequency bandwidth of radio signal. If correction is not achieved, the cause might be either a damaged gradient coil or an abnormal current passing through the gradient coil.

\section{RF inhomogeneity ${ }^{15}$}

Variation in intensity across the image may be due to the failure of the
RF coil, non-uniform B1 field, nonuniform sensitivity of the receive only coil (spaces between wire in the coil, uneven distribution of wire), or presence of non-ferromagnetic material in the imaged object.

\section{Asymmetrical brightness ${ }^{14}$}

There is a uniform decrease in signal intensity along the frequencyencoding axis. Signal drop-off is due to filters that are too tight about the signal band. Some of the signal generated by the imaged section is, thereby, inappropriately rejected. A similar artefact may be caused by non-uniformity in slice thickness.

\section{RF noise ${ }^{14,15}$}

RF pulses and precessional frequencies of MRI instruments occupy the same frequency bandwidth as common sources such as TV, radio, fluorescent lights and computers.

Stray RF signals can cause various artefacts. Narrow-band noise is projected perpendicular to the frequency-encoding direction. Broadband noise disrupts the image over a much larger area. Appropriate site planning, proper installation and RF shielding (Faraday cage) eliminate stray RF interference.

Zero line and star artefacts. A bright linear signal in a dashed pattern that decreases in intensity across the screen and can occur as a line or star pattern, depending on the position of the patient in the 'phase-frequency space'.

Zero line and star artefacts are due to system noise or any cause of RF pollution within the room (Faraday cage). If this pattern persists, check for sources of system noise such as bad electronics or alternating current line noise, loose connections to surface coils, or any source of RF pollution. If a star pattern is encountered, the manufacturer should readjust the system software so that the image is moved off the zero point.

Stars/zippers. Although less common, stars/zippers are bands through the image centre due to an imperfect Faraday cage, with RF pollution in, but originating from outside, the cage.

Residual free induction decay stimulated echo also causes stars/zippers.

\section{RF tip angle inhomogene- ity $^{15}$}

These are patchy areas of increased or decreased signal intensity. This artefact is produced by variations in $\mathrm{RF}$ energy required to tip protons 90 or 180 degrees within the selected slice volume.

\section{Bounce point artefact ${ }^{15}$}

Absence of signal from tissues of a particular $\mathrm{T} 1$ value is a consequence of magnitude sensitive reconstruction in inversion recovery imaging. When the chosen T1 equals $69 \%$ of the $\mathrm{T} 1$ value of a particular tissue, a bounce point artefact occurs.

Use phase-sensitive reconstruction inversion recovery techniques.

\section{Surface coil artefacts (attenuation of signal) ${ }^{15}$}

Close to the surface coil the signals are very strong resulting in a very intense image signal (Fig. 10). Further from the coil the signal strength drops rapidly due to the attenuation with a loss of image brightness and significant shading 


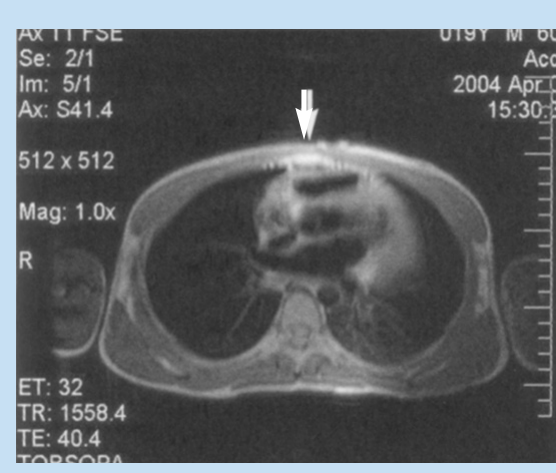

Fig. 10. Surface coil artefact: high signal at anterior thoracic wall adjacent to surface coil.

to the uniformity. Surface coil sensitivity intensifies problems related to RF attenuation and RF mismatching.

\section{Slice-to-slice interference ${ }^{14}$}

Non-uniform RF energy received by adjacent slices during a multi-slice acquisition is due to cross-excitation of adjacent slices with contrast loss in reconstructed images (Fig. 11).

To overcome these interference artefacts, include the acquisition of two independent sets of gapped multi-slice images, subsequently reordered during display of the full

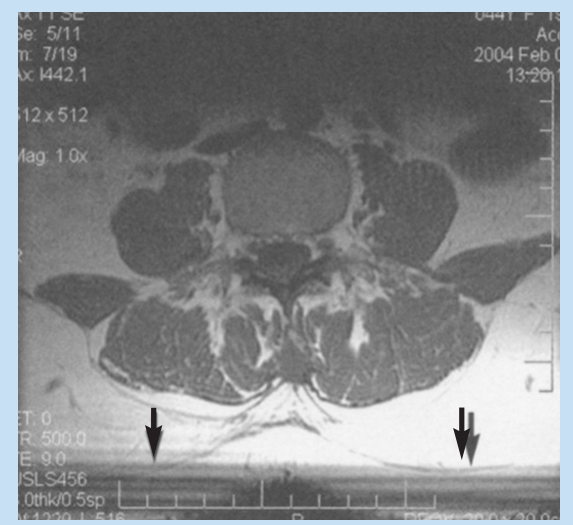

Fig. 11. Slice-to-slice interference (T1 axial study of lumbar vertebrae).

image set.

\section{References}

1. Elster AD, Burdette JH. Questions \& Answers in Magnetic Resonance Imaging. 2nd ed. St. Louis: Mosby, 2001.

2. Huda W, Slone RM. Review of Radiologic Physics. Philadelphia: Lippincott, Williams \& Wilkins, 1995.

3. Nguyen TD, Ding G, Watts R, Wang Yi. Optimization of view ordering for motion artifact suppression. Magn Reson Imaging 2001; 19: 951-957.

4. Suh JS, Jeong EK, Shin KH, et al. Minimizing artifacts caused by metallic implants at MR imaging: experimental and clinical studies. Am J Roentgenol 1998; 171: 1207-1213.

5. Chang SD, Lee MJ, Munk PL, Janzen DL, MacKay A, Xiang QS. MRI of spinal hardware: comparison of conventional T1-weighted sequence with a new metal artifact reduction sequence. Skeletal Radiol 2001; 30: 213-218.

6. Viano AM, Gronemeyer SA, Haliloglu M, Hoffer FA. Improved MR imaging for patients with metallic implants. Magn Reson Imaging 2000; 18: 287-295.

7. Smith RC, Lange RC, McCarthy SM. Chemical shift artifact: dependence on shape and orientation of the lipid-water interface. Radiology 1991; 181: 225-229.

8. Elgavish RA, Twieg DB. Improved depiction of small anatomic structures in MR images using Gaussian-weighted spirals and zero-filled interpolation. Magn Reson Imaging 2003; 21: 103112.

9. Lee SJ. An improved method for reduction of truncation artifact in magnetic resonance imaging. Proceedings of SPIE: Applications of Digital Image Processing 1998; 4360: 587-598.

10. Archibald R, Gelb A. A method to reduce the Gibbs ringing artifact in MRI scans while keeping tissue boundary integrity. IEEE Trans Med Imaging 2002; 21: 305-319.

11. Bakir T, Reeves SJ. A filter design method for minimizing ringing in a region of interest in MR spectroscopic images. IEEE Trans Med Imaging 2000; 19: 585-600.

12. Schultz RR, Stevenson RL. A Bayesian approach to image expansion for improved definition. IEEE Trans Med Imaging 1994; 3: 233-242.

13. Holden M, Breeuwer MM, McLeish K, Hawkes DJ, Deevil SF, Hill DL. Sources and correction of higher-order geometrical distortion for serial MR brain imaging. Proceedings of SPIE 2001; 4322: 69-78.

14. Bushberg JT, Seibert JA, Leidholdt jun. EM, Boone JM. The Essential Physics of Medical Imaging. Baltimore: Williams and Wilkins, 1994.

15. Pusey E, Lufkin RB, Brown RK, et al. Magnetic resonance imaging artifacts: mechanism and clinical significance. Radiographics 1986; 6: 891911. 